\title{
Failure of Mitral Valve Repair: Partial Detachment of Valvular Ring by 3D Transesophageal Echocardiography Reconstruction
}

\author{
Alessandro Malagoli, M.D., Francesca Bursi, M.D., and Maria Grazia Modena, M.D., F.E.S.C. \\ Department of Cardiology, Policlinico Hospital, Modena and Reggio Emilia University, \\ Modena, Italy
}

(ECHOCARDIOGRAPHY, Volume 26, January 2009)

$3 D$ transesophageal echocardiography, detachment of valvular ring, mitral valve

A 75-year-old man with systemic hypertension and permanent atrial fibrillation was referred to our institution for an echocardiographic examination because of mild exertional dyspnea. Two years prior, he underwent mitral valve quadrangular resection and anuloplasty because of severe mitral regurgitation due to flail of the posterior leaflet. By transthoracic echocardiography we observed recurrence of severe mitral regurgitation. Twodimensional (2D) transesophageal echocardiography (TEE) and three-dimensional (3D) TEE acquisitions were obtained with the same ultrasound unit which incorporates 3D data acquisition software. Off-line postprocessing and 3D reconstruction were performed through a dedicated system (fourSight ${ }^{\mathrm{TM}}$ TEE View, Siemens, Mountain View, CA, USA). The 2D TEE exam showed residual prolapse and flail of the posterior leaflet (Fig. 1, panel A) with multiple and complex (perianular and intranular) regurgitant jets (Fig. 1, panel C). By the 3D TEE reconstruction, we were able to confirm the posterior leaflet prolapse and in addition we observed a large anterior orifice due to partial detachment of the valvular ring. Also we were able to measure the size of three anatomic orifice areas (AOAs) (Fig. 1, panel D). The patient underwent successful mitral valve replacement and the pathology specimen

Conflicts of interest: None declared.

Address for correspondence and reprint requests: Alessandro Malagoli, M.D., Department of Cardiology, Policlinico Hospital, Modena and Reggio Emilia University, Modena, Italy. Fax: +39-059-4224323; E-mail: ale.mala@tiscali.it confirmed the 3D echocardiographic findings (Fig. 1, panel B).

There are little data on quantification of the AOAs by $3 \mathrm{D}$ transesophageal or transtoracic echocardiography mitral valve reconstructions ${ }^{1-3}$ and all about intravalvular jet. To the best of our knowledge, this is the first report of a partial detachment of valvular ring by $3 \mathrm{D}$ TEE reconstruction.

\section{References}

1. Breburda CS, Griffin BP, Pu M, et al: Threedimensional echocardiographic planimetry of maximal regurgitant orifice area in myxomatous mitral regurgitation: Intraoperative comparison with proximal flow convergence. J Am Coll Cardiol 1998;32(2):432437.

2. Lange A, Palka P, Donnelly J, et al: Quantification of mitral regurgitation orifice area by 3-dimensional echocardiography: Comparison with effective regurgitant orifice area by PISA method and proximal regurgitant jet diameter. Int $J$ Cardiol 2002;86(1):8798.

3. Kuppahally SS, Paloma A, Craig Miller D, et al: Multiplanar visualization in 3D transthoracic echocardiography for precise delineation of mitral valve pathology. Echocardiography 2008;25(1):84-87.

\section{Supplementary Material}

The following supplementary material is available for this article online:

Video clip 1: 3D TEE reconstruction of the mitral valve in the systolic phase (left atrial view).

Video clip 2: 3D TEE reconstruction of the mitral valve in the systolic phase (left atrial view) with more filter than Video clip 1. 


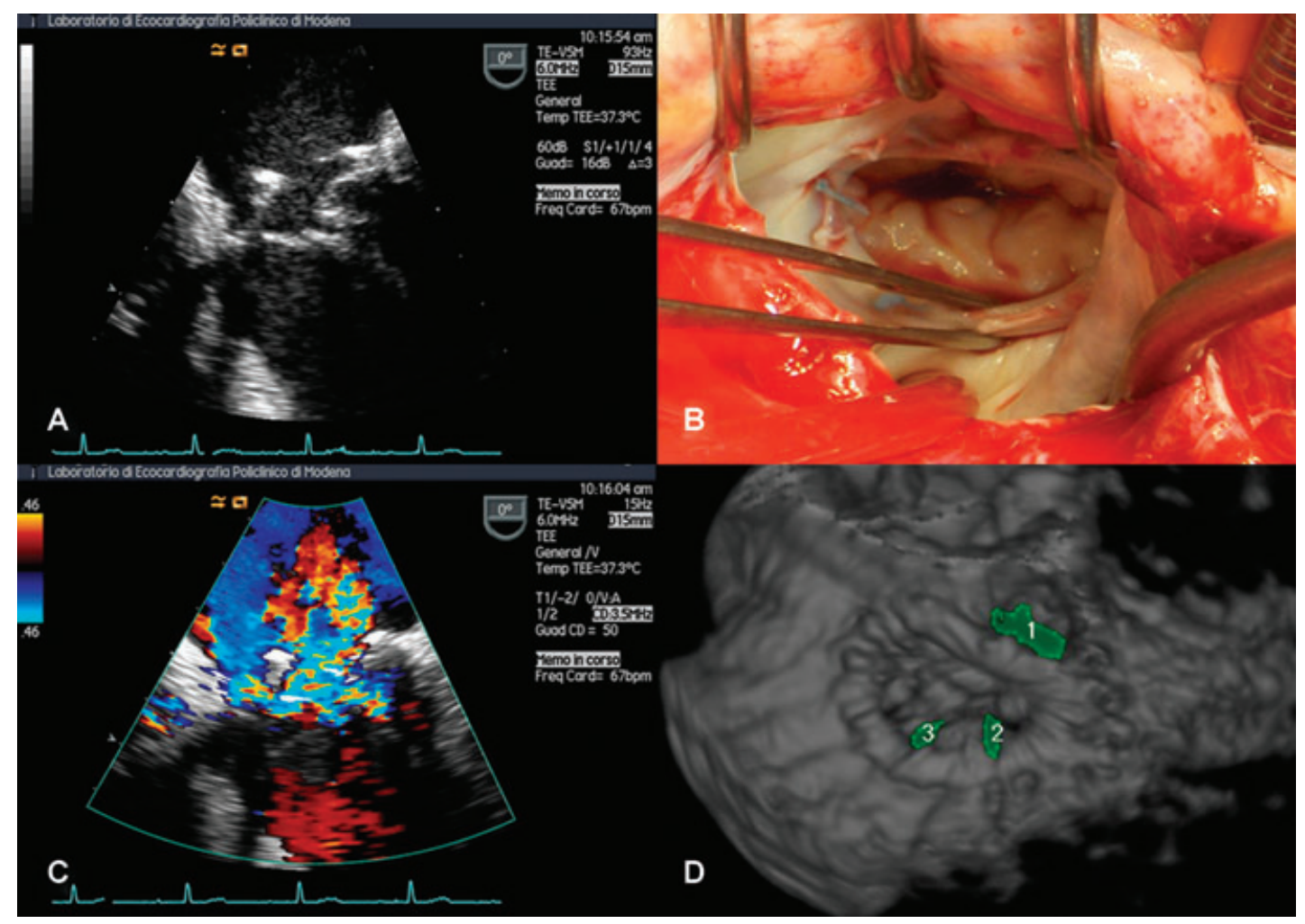

Figure 1. A. 2D TEE 0 grade (zoom): typical four chambers showing a valvular ring in diastolic phase and residual prolapse and flail of the posterior leaflet. B. Surgical view (left atrial view). Partial detachment of a valvular ring on the anterior leaflet (upper leaflet) of the mitral valve. C. $2 D$ TEE 0 grade (zoom) with color Doppler showing multiple regurgitant jets (perianular and intranular). D. $3 D$ TEE reconstruction of the mitral valve in the systolic phase (left atrial view). By $3 D$ TEE reconstruction we were able to diagnose and measure the size of three AOAs [quantification: (1) $0.2 \mathrm{~cm}^{2}$; (2) $0.1 \mathrm{~cm}^{2}$; (3) $0.1 \mathrm{~cm}^{2}$ ] (see Video clips 1-4).

Video clip 3: 3D TEE reconstruction with the scalpel tool (left atrial view in the diastolic phase). It is possible to recognize two orifices. Down-left is the physiological one due to the opening of the mitral valve; upright is the pathological one due to the partial detachment of the valvular ring.
Video clip 4: 3D TEE reconstruction with the scalpel tool (left ventricular view in the diastolic phase). It is possible to recognize two orifices. Down-left is the physiological one due to the opening of the mitral valve. Upright is the pathological one due to the partial detachment of the valvular ring. 